\title{
Comprehensive Risk Evaluation in the Phase of Setting Information System Project
}

\author{
Gao Yinmin \\ Business College of Beijing Union University, Beijing 100025,China
}

\begin{abstract}
The IS has the characteristic of high risk and high profit. It makes the IS project- setting risk evaluation system becoming an urgent research, and getting more and more attention. However, the development of the IS is a complicated system engineering, project-setting risk will involve with many factors, therefore, it has become a difficulty of the management field. Based on the code for comprehensive risk evaluation design of the IS project- setting risk and suggestions from specialists, an index system has been established for the evaluation of the IS project- setting risk. The first hierarchy indexes consist of four parts, i.e., inherent management risk, technology risk, user involvement and training risk and Implementing risk, each of which in turn has its own subindexes. In order to indicate the importance of each index to the project-setting decision of IS, each weight is determined according to the Analytical Hierarchy Process. On the basis of fuzzy theory, a comprehensive assessment model for comprehensive risk evaluation design of the IS project- setting risk is established. The evaluation result is given according to the principle of greatest membership. In the end, we cite an example to illustrate the suggested approach. The evaluation results agree with the real situation pretty well, representing that the model can be used as the scientific basis for powerful supporting for the project-setting decision of IS .
\end{abstract}

Key words: information system; risk evaluation;index system; evaluation method; fuzzy comprehensive evaluation

\section{Introduction}

Failing to understand and manage Information System Project risk can lead to a variety of problems

GAO Yinmin is with the Department of e-business Business College of Beijing Union University, Beijing China (phone:8601065086749; fax: 86-010-65940655; e-mail: Cgaoyinmin@126.com).

including cost and schedule overruns, unmet user requirements, and the production of systems that are not used or do not deliver business value.A simple definition of "risk" is a problem that hasn't happened yet but could cause some loss or threaten the success of your project if it did (Wiegers, 1998). A number of research studies have investigated the issue of the relative importance of various risks in software development projects and have attempted to classify them in various ways. Much has been written about the causes of information systems project failures. Poor technical methods is only one of

the causes, and this cause is relatively minor in comparison to larger issues, such as failures in communications and ineffective leadership.Managing the risk associated with them becomes a critical area of concern [1, 2]. Advocates of IS risk management argue that identifying and analyzing threats to success allows actions to be taken to reduce the chance of failure. Articles have stressed the importance of empirically categorizing the sources and types of risks associated with software development projects [3] Unfortunately, despite these recommendations there are relatively few tools available to help project managers identify and categorize risk factors in order to develop effective strategies. While various risk checklists (e.g., the "top-10" list of risk factors described by and frameworks [4] have been proposed, the underlying dimensions of the software project risk construct and their influence on a project remain largely unexplored.

With systems that involve the use of new client-server technology, it is often critical to acquire external expertise,including vendor support, to facilitate successful implementation.Also, the costs of training and support are often under-estimated,and these costs may be many times greater than originally anticipated. Client-server implementations often bring"surprises" with respect to cost, because of the costs of decentralized servers, systems integration software, technical support, and software updates and version control. In actuality, the total cost of a client server implementation can be three to six times greater than for a comparable mainframe-based system.Even though there are great cost reductions possible through moving off the mainframe, the costs of learning the new technology and of acquiring technical support are substantial.

By applying the principle and method of fuzzy comprehensive evaluation, the article establishes evaluation system for comprehensive risk evaluation design of the setting IS project(shortened form SISP) risk which is suitable for the present status of setting IS project decision in our country. I intend to provide a relatively scientific method for SISPin our country through my preliminary exploration. I also expect there can be more experts to concern about Information System Project risk evaluation, so as to make contribution for enhancing manage Information System Project risk.

\section{Index System Design of the Evaluation of the SISP Risk.}

The first step of the evaluation is to construct the evaluating index system scientifically, which is the key to success of evaluation. The evaluation of the IS project- setting risk is a complex system with a large number of functional parameters. The overall risk level is determined by the parameters, that is, by

$$
T R L=f(R M L, R T L, R U L, \cdots)
$$


Wherein $T R L=$ total SISP risk level; $R M D=$ risk level with respect to management risk; $R T L=$ risk level with respect to technology risk, $R U L=$ safety level with respect to user involvement and training risk, and so on.

On the basis of the code for the "top-10" list of risk factors described by and frameworks study, experts' investigation and also referring other papers ${ }^{[4,5]}$, an index system has been established for The evaluation of the IS project- setting risk The first hierarchy indexes consist of four parts: inherent management risk, technology risk, user involvement and training risk and Implementing risk each of which has its own sub-indexes. Index system is shown in Fig.1.

\section{The Weighting of the Attribute for Evaluation}

Not all risk criteria have equal importance. Parameter weights serve to express the importance of each criteria compared with the others. Hence the assignment of weights is a key component of multi-attribute evaluation. The accuracy of the evaluation depends on the accuracy of the weighting assigned to each criterion, which can be determined by setting up an expert panel and synthesizing - for example, by approximate reasoning - the average ranking of the criteria for evaluation purposes. Since a large number of criteria are needed and it is difficult to obtain a consistent result from each of the experts, a systematic method is required to rank the criteria.

The Analytical Hierarchy Process (AHP), developed by Saaty ${ }^{[7]}$, is one of the methods used in multi-criteria decisionmaking and may be employed to assist individuals and groups in ranking the fire safety attributes. By incorporating both subjective and objective data into a logical hierarchical framework, AHP provides decision-makers with an intuitive approach to evaluating the importance of every element of a decision through pairwise comparison. The AHP is best suited for multi-criteria problems for which it is not possible to accurately quantify the impact of each of the alternatives. For this reason, the AHP is effective for ranking each fire safety attribute in The evaluation of the IS project- setting risk because the attributes that involve people interacting are difficult to quantify The paper uses AHP to calculate the weight of each index. The results are shown as followings:

$$
\begin{aligned}
W & =\left(W_{1}, W_{2}, W_{3}, W_{4}\right)=(0.28,0.33,0.19,0.20) \\
W_{1} & =\left(W_{11}, W_{12}, W_{13}, W_{14}, W_{15}\right) \\
& =(0.23,0.12,0.29,0.21,0.15) \\
W_{2} & =\left(W_{21}, W_{22}, W_{23}, W_{24}, W_{25}, W_{26}, W_{27}\right) \\
& =(0.11,0.25,0.15,0.16,0.12,0.11,0.10) \\
W_{3} & =\left(W_{31}, W_{32}, W_{33}, W_{34}, W_{35}\right) \\
& =(0.28,0.21,0.20,0.17,0.14)
\end{aligned}
$$

$$
\begin{aligned}
W_{4}= & \left(W_{41}, W_{42}, W_{43}, W_{44}, W_{45}\right) \\
& =(0.24,0.24,0.21,0.20,0.11)
\end{aligned}
$$

\section{Fuzzy Comprehensive Evaluation Model for the comprehensive evaluation Risk Degree of ISSP}

The comprehensive evaluation risk degree of ISSP involves many factors. What is more, there are abounding uncertainty factors and dynamic variable with high fuzziness. An assessment model for the comprehensive evaluation risk degree of ISSP is established by applying fuzzy mathematics theory in this paper.

\subsection{Simple Fuzzy Comprehensive Evaluation}

Index set is $X=\left\{x_{1}, x_{2}, \cdots, x_{n}\right\}$. Evaluation set is $Y=\left\{y_{1}, y_{2}, \cdots, y_{m}\right\}$ (in the paper, $\mathrm{m}=4, \mathrm{y}_{1}=$ very risk, $\mathrm{y}_{2}=$ risk, $\mathrm{y}_{3}=$ basically risk, $\mathrm{y}_{4}=$ low risk ). Fuzzy relation $R$ between index and evaluation represents the possibility of making all kinds of evaluation on various indexes $x_{i}$. For example, $r_{i j}$ represents the possibility of making evaluation $y_{j}$ on $x_{i} . w$ is a weight distribution. $w=\left(w_{1}, w_{2}, \cdots, w_{n}\right)$ represents the significance of each index in evaluation. For example, $w_{i}$ represents the significant value of factor $x_{i}$ in evaluation. The result of evaluation is fuzzy sets $B=\left(b_{1}, b_{2}, \cdots, b_{m}\right)$, which represents the degree of subordination of making all kinds of evaluation. For example, $b_{j}$ represent the degree of subordination of comprehensive evaluation for $y_{j}$.

\subsection{Multi-hierarchy Fuzzy Comprehensive Evaluation}

There are many factors affecting the comprehensive evaluation Risk Degree of ISSP,, and their structure is very complex. In the above research, we have already set up the index system of evaluation according to the internal relations of each factor. Therefore, we set up the multi-hierarchy fuzzy comprehensive evaluation model.

$$
B=\left(b_{1}, b_{2}, \cdots b_{m}\right)=\left(w_{1}, w_{2}, \cdots, w_{n}\right) \circ\left[\begin{array}{ccc}
r_{11} & \cdots & r_{1 m} \\
\vdots & \vdots & \vdots \\
r_{n 1} & \cdots & r_{n m}
\end{array}\right]
$$

Multi-hierarchy fuzzy comprehensive evaluation is implemented in the following steps :

(1) Separate the index set

$$
\begin{aligned}
& X=\left\{X_{1}, X_{2}, \cdots, X_{n}\right\} \text { into } s \text { subsets } \\
& X_{i}=\left\{x_{i_{1}}, x_{i_{2}}, \cdots, x_{i_{n_{i}}}\right\}, i=1,2, \cdots, s
\end{aligned}
$$

Which meets the conditions of 


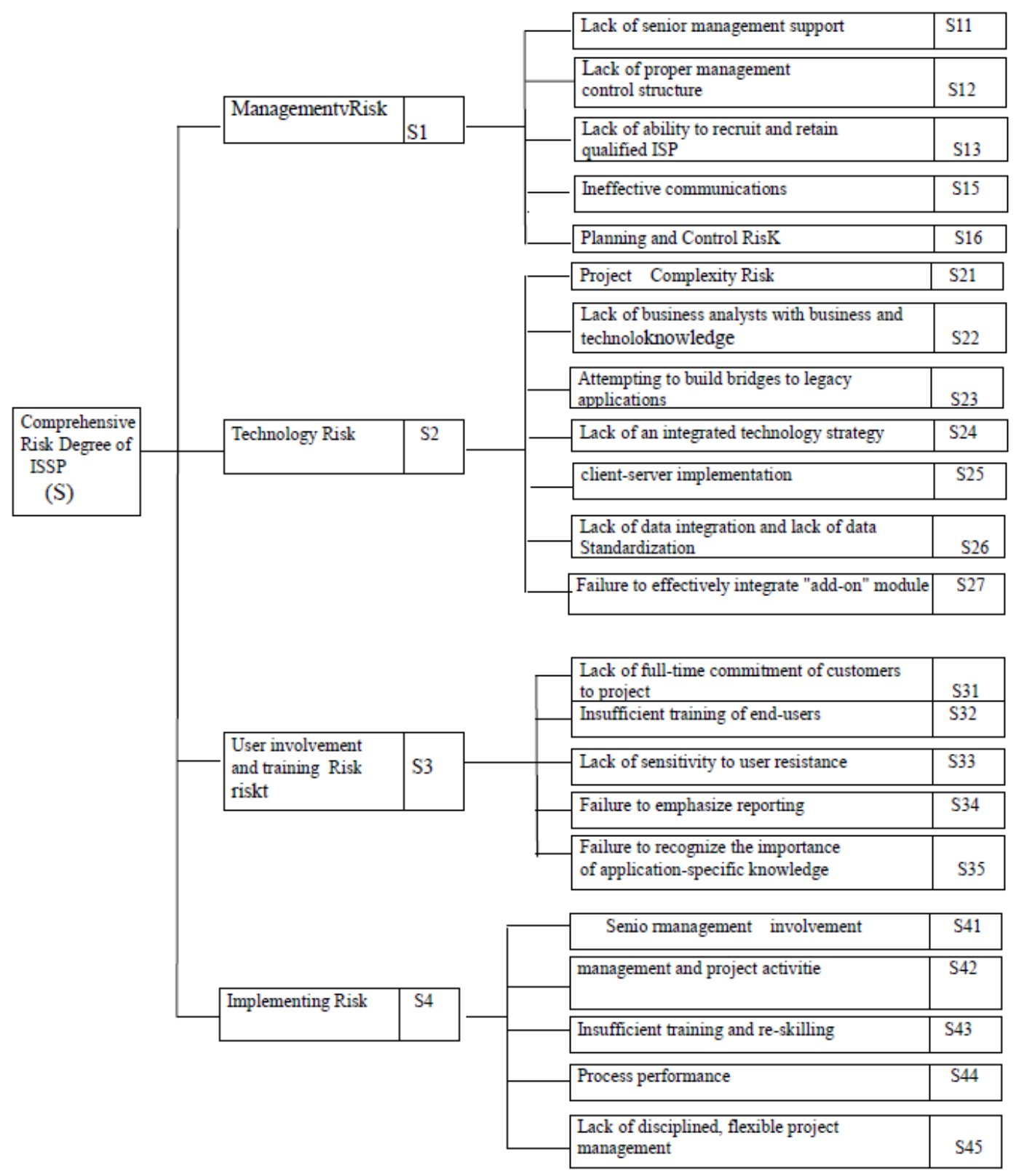

Fig.1 Index system of Comprehensive evaluation for ISSP

$$
\sum_{i=1}^{s} n_{i}=n ; \bigcup_{i=1} X_{i}=X ; X_{i} \cap X_{j}=\Phi, i \neq j
$$

(2) Implement comprehensive evaluation on each $X_{i}$ with above-mentioned simple fuzzy comprehensive evaluation model, respectively.

Set up remark set $Y=\left\{y_{1}, y_{2}, \cdots, y_{m}\right\}$. The weight distribution of each factor in $x_{i}$ is:

$$
w_{i}=\left(w_{i_{1}}, w_{i_{2}}, \cdots, w_{i_{n_{i}}}\right)
$$

$$
\sum_{j=1}^{n_{i}} w_{i j}=1 w_{i j} \geq 0, j=1,2, \cdots, n_{i}
$$

If the single-factor assessment matrix of $x_{i}$ is $R_{i}$, then the first order comprehensive evaluation of the index is:

$$
\begin{gathered}
B_{i}=w_{i} \circ R_{i}=\left(b_{i 1}, b_{i 2}, \cdots, b_{i m}\right), \\
i=1,2, \cdots, s
\end{gathered}
$$


(3) If each $x_{i}$ is considered as one index and the single-factor evaluation result is $b_{i}$, then the single index evaluation matrix of $X=\left\{x_{1}, x_{2}, \cdots, x_{s}\right\}$ will be $R$

$$
R=\left[\begin{array}{c}
B_{1} \\
B_{2} \\
\vdots \\
B_{s}
\end{array}\right]=\left[\begin{array}{cccc}
b_{11} & b_{12} & \cdots & b_{1 m} \\
b_{21} & b_{22} & \cdots & b_{2 m} \\
\vdots & \vdots & \vdots & \vdots \\
b_{S 1} & b_{S 2} & \cdots & b_{S m}
\end{array}\right]
$$

As a part of $X$, each $X_{i}$ reflects a kind of attribution of $X$. In this way, the weight distribution $W=\left(w_{1}^{*}, w_{2}^{*}, \cdots, w_{S}^{*}\right)$ can be presented in terms of their importance. is:

So, the second order comprehensive evaluation vector

$$
B=W \circ R=\left(b_{1}, b_{2}, \cdots, b_{m}\right)
$$

If the first order index set $X_{i}, i=1,2, \cdots s$, still contain more factors, $X_{i}$ can be separated into lesser sets. So the three-stage model or four-stage model can be obtained in the same way.

\subsection{The Determination of Logical Operation Method}

The logical operator of the above-mentioned fuzzy comprehensive evaluation model $B=w \circ R$ is " 。".

This paper adapts the weighted average model :

$$
b_{j}=\sum_{k=1}^{n} w_{k} \cdot y_{k j}
$$

Compared with other common models, such as $(\vee, \wedge)$ Model and $(\vee, \bullet)$ Model, the model can furthest reserve the evaluation opinions of all those specialist with least information distortion.

\subsection{The Criteria for Evaluation.}

In this paper, the result is obtained on the principle of greatest membership. That is to say, if $b_{j}=\max \left\{b_{1}, b_{2}, \cdots, b_{m}\right\}$, and then the comprehensive evaluation result will be $y_{j}$.

\section{An Example of Application}

Provided that 10 experts are invited to participate in the comprehensive evaluation Risk Degree of ISSP, the singlefactor evaluation matrix of inherent management risk, technology risk, user involvement and training risk and Implementing risk is represented respectively as follows:

$$
\begin{aligned}
R_{1} & =\left[\begin{array}{llll}
0.00 & 0.70 & 0.30 & 0.00 \\
0.10 & 0.60 & 0.30 & 0.00 \\
0.00 & 0.90 & 0.10 & 0.00 \\
0.90 & 0.10 & 0.00 & 0.00 \\
0.50 & 0.50 & 0.00 & 0.00
\end{array}\right] \\
R_{3} & =\left[\begin{array}{llll}
0.00 & 0.70 & 0.20 & 0.10 \\
0.10 & 0.60 & 0.10 & 0.20 \\
0.00 & 0.70 & 0.20 & 0.10 \\
0.10 & 0.30 & 0.30 & 0.30 \\
0.10 & 0.60 & 0.20 & 0.10
\end{array}\right]
\end{aligned}
$$

$$
R_{2}=\left[\begin{array}{llll}
0.10 & 0.40 & 0.50 & 0.00 \\
0.00 & 0.20 & 0.70 & 0.10 \\
0.80 & 0.20 & 0.00 & 0.00 \\
0.00 & 0.60 & 0.30 & 0.10 \\
0.10 & 0.80 & 0.10 & 0.00 \\
0.00 & 0.50 & 0.50 & 0.00 \\
0.40 & 0.60 & 0.00 & 0.00
\end{array}\right]
$$

$$
R_{4}=\left[\begin{array}{llll}
0.00 & 0.70 & 0.30 & 0.00 \\
0.80 & 0.20 & 0.00 & 0.00 \\
0.00 & 0.70 & 0.20 & 0.10 \\
0.40 & 0.20 & 0.40 & 0.00 \\
0.10 & 0.70 & 0.20 & 0.00
\end{array}\right]
$$

So the result of simple fuzzy comprehensive assessment can be obtained as following:

$$
\begin{aligned}
& B_{1}=W_{1} \circ R_{1}=(0.276,0.590,0.134,0.000) \\
& B_{2}=W_{2} \circ R_{2}=(0.184,0.431,0.345,0.040 \\
& B_{3}=W_{3} \circ R_{3}=(0.052,0.597,0.196,0.155) \\
& B_{4}=W_{4} \circ R_{4}=(0.283,0.480,0.216,0.021)
\end{aligned}
$$

The fuzzy matrix of multi-factors assessment can be obtained as following:

$$
R=\left[\begin{array}{l}
B_{1} \\
B_{2} \\
B_{3} \\
B_{4}
\end{array}\right]=\left[\begin{array}{llll}
0.276 & 0.590 & 0.134 & 0.000 \\
0.184 & 0.431 & 0.345 & 0.040 \\
0.052 & 0.597 & 0.196 & 0.155 \\
0.283 & 0.480 & 0.216 & 0.021
\end{array}\right]
$$

Then the obtained result of fuzzy comprehensive assessment is: 


$$
B=W \circ R=\left(\begin{array}{llll}
0.204 & 0.517 & 0.232 & 0.047
\end{array}\right)
$$

According to the principle of greatest membership (the greatest value is 0.517 ), we can draw a conclusion that the setting IS decision is the characteristic of high risk .

\section{Conclusions}

With the help of the evaluation model established in the paper, the comprehensive risk evaluation design of the IS project- setting risk a can be evaluated with higher accuracy. The result of evaluation can be used as the scientific basis for setting IS decision,carrying out IS management in a reasonable way.

This paper outlines the framework of fuzzy comprehensive evaluation for setting IS decision. Further studies are needed to clarify the details of setting up the system, including forming expert panels to formulate the numerical values of the attributes' weights, carrying out sensitivity analysis, determining the most suitable method for evaluation, and verifying the accuracy of the method. The rationality of each index and its weight should undergo continuous examination through practical application. We shall adjust index and its weight flexibly to make it more reasonable to meet the requirements of practical application. The precise definition and quantification of each index is not stated in the paper. We will do it in the next research work.

\section{References}

[1] R. Kumar, Managing risks in IT projects: an optionsperspective, Information and Management 40, 2002, pp. 63-74.

[2] J.S. Osmundson, J.B. Michael, M.J. Machniak, M.A. Grossman, Quality management metrics for software development,Information and Management 40, 2003, pp. 799-812.

[3] P.P. Tallon, et al. Using Real Options Analysis for Evaluating Uncertain Investments inInformation Technology[A]: Insights From The ICIS 2001 Debate, Communications of the Association for Information Systems 2002, 9: 136 167.

[4] M. Keil, P. Cule, K. Lyytinen, R. Schmidt, A framework foridentifying software project risks, Communications of theACM 41 (11), 1998, pp. 76-83.

[5] Walsh, K.R. \& Schneider, H. The role of motivation and risk behaviors in software development success. Information Research, (2002) 7 (3).

[6] Keil, Mark; Cule, Paul E.; Lyytinen, Kalle; and Schmidt,Roy C., "A Framework for identifying software project risks," Communications of the ACM, V. 41, No. 11, Nov.1998, pp. 76-83.

[7] Thomas L.Saaty and Luis G.Vargas. Models, Methods, Concepts \& Applications of the Analytic Hierarchy Process. Boston:Kluwer Academic Publishers. 2001:1 13 